preasure $0.6,1 \cdot 0$ and $1.3 \mathrm{~mm}$. mercury. There was a decrease in noise output with increase in shunt capacity, the decrease being most marked for the highest two frequencies.

Since the anode of the tube is near the spectrograph slit, the positive column near the anode contributes most to the intensity of $\lambda 1215 \mathrm{~A}$. Instability of the discharge makes observations with reversed polarity difficult ; but it appears that when the cathode is nearest the slit the intensity is not very different.

It appears that, together with the disappearance of the striations and a decrease in radio-frequency noise, there is a reduction in the population of electrons having sufficient energy to excite hydrogen atoms. Large numbers of electrons with about 11 volts energy have been found in the striated column ${ }^{2}$, and it is assumed that $\lambda 1215 \mathrm{~A}$. results from the excitation of dissociated atoms. The intensity of the molecular lines, which have about the same excitation energy, should then decrease at the same time as the atomic lines. A connexion between the radiofrequency noise intensity and the energy distribution among the electrons, which is strongly suggested by our results, requires further elucidation.

We desire to acknowledge the financial assistance provided by the Research Grants Committee of the University of Western Australia and the help of Miss N. E. White in making some of the measurements. We are also indebted to Dr. R. L. F. Boyd, of University College, London, for discussion of these results.

S. E. WIILIAMS

Department of Physics,

University of Western Australia.

Oct. 15.

${ }^{1}$ Bolton and Williams, Brit. J. App. Phys., 4, 6 (1953).

: Boyd, R. L. (private communication).

\section{Shadow Microscopy for Measurement of Height}

Tolansky has described ${ }^{2}$ an elegant method for observing, with a microscope, features of the threedimensional profiles of particles and other small objects. For a particle of known geometry, it is correct to say that the height can be measured accurately

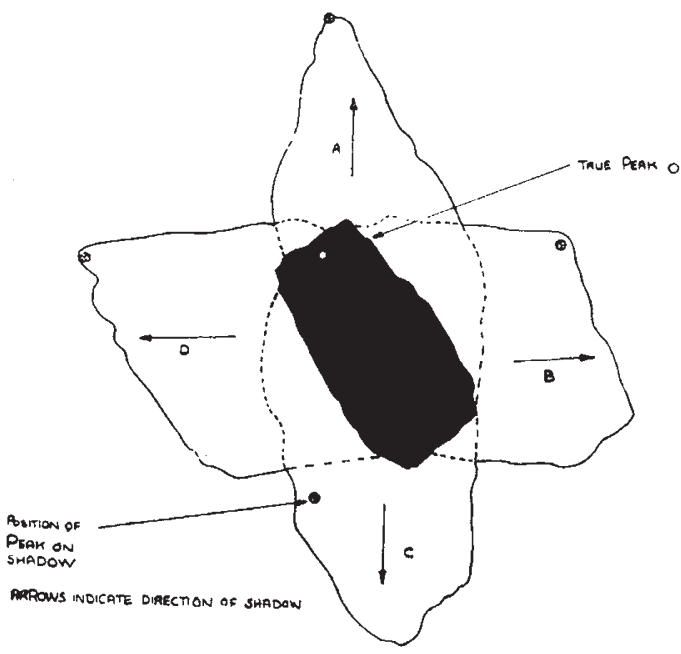

from the length of a shadow, which may be produced by Tolansky's method or by the usuel metal shadowcasting technique. For a particle of irregular shape, however, such as a dust particle, height can only be determined approximately, for the following reasons, which are illustrated in the diagram: (i) the peak may not be in a position to throw the longest shadow; (ii) the plan position of the peak is not known, and consequentiy neither is that of the base line frnm which to measure the length of the shadow.

This problem has been investigated on a macroscopic scale, with lumps of coal and smooth weathered stones, 4-8 cm. in size. It was found that the best measure of height is given by $S \sin \theta$, where $\theta$ is the shadow angle and $S$ is the distance from the mid-length of a particle, measured in the direction of shadowing, to the tip of the shadow. The percentage standard deviation, $\sigma$, of the estimate of height given by $S \sin \theta$ is well represented for both series, over a range of $\theta$ from $10^{\circ}$ to $30^{\circ}$, by

$$
\sigma=20(\sin \theta)^{0.75} \text {. }
$$

Details of this investigation will be published elsewhere.

Suffield Experimental Station,

H. H. WATSON

Defence Research Board,

Ralston, Alberta,

Canada.

Oct. 21.

${ }^{1}$ Tolansky, S., Nature, 171, 364 (1953).

\section{Two Half-shade Devices for Optical Polarizing Instruments}

The development of interference microscopes of the double-refracting type has stimulated a renewed interest in eyepieces having at their focal planes sensitive birefringent devices for increasing the accuracy with which forms of polarization can be determined. These devices usually employ a boundary or boundaries formed by one or more birefringent plate elements viewed through an anslyser, and the difficulty arises of making boundaries sufficiently precise for the maximum sensitivity to be achieved. The present communication is a brief account of two rather promising solutions to this problem which have recently been investigated here.

In the case of the first and rather more elaborate solution, the boundary is artificially formed by doublerefraction of the virtual image of a straight wire located at the focal plane of a positive eyepiece. The double-refraction is adjusted to produce a lateral displacement which is precisely the same as the diameter of the wire, resulting in the formation of a very perfect boundary between the two images of the wire. Since the two images are formed by doublerefraction, they are polarized in mutually perpendicular planes and their intensity relationship is therefore a function of the polarization azimuth of the incident light, the intensity ratio being $\cos ^{2} \theta / \sin ^{2} \theta$, where $\theta$ is the angle which the incident vibration azimuth makes with the vibration direction of one of the two images. An analyser is consequently unessential; but the addition of one, so orientated that its vibration azimuth makes unequal angles with the mutually perpendicular vibration azimuths of the two wire images, results in an enhanced sensitivity which is very approximately proportional to the angular inequality. 